\title{
Transformationen entstehen im Prozess: Transnationale Feminismen zwischen Dekolonisierung, imperialen Verwobenheiten und der Suche nach neuen Solidaritäten
}

\author{
Uta Ruppert und Tanja Scheiterbauer im Gespräch \\ mit Nikita Dhawan, Esther Franke, Radwa Khaled \\ und Christa Wichterich
}

Transnationale Feminismen sind längst nicht mehr das, was im 20. Jahrhundert mit diesem Begriff zusammengefasst wurde. Die gesamte Bandbreite der Theorien und sozialen Praxen von nationale Grenzen und politische Handlungsebenen überschreitenden Feminismen haben in den letzten (mehr als) 25 Jahren die Welt in vielerlei Hinsicht verändert, während sie selbst sich immer auch im Verhältnis zu den Veränderungen der Weltpolitik gewandelt haben. Dabei war das, was unter transnationalen Feminismen zu verstehen ist, "innerfeministisch" stets umstritten und durchaus auch umkämpft. Die mittlerweile fast als paradigmatisch anzusehenden Definitionen aus den 1990er Jahren, die den Rahmen der theoretischen Diskussion über transnationale Feminismen absteckten, reichen von der materialistischen, in der Black Feminist Theory verwurzelten Perspektive von Jacqui Alexander und Chandra Mohanty (1997), die Fragen der Positionalität mit jenen nach materialer Gerechtigkeit verknüpft, über die poststrukturalistisch geprägte Position von Inderpal Grewal und Caren Kaplan (1994), die Dezentrierungen von Kategorien und (räumlichen) Konzepten einfordert, bis zum strikt institutionalistischen, auf Organisations- und Verhandlungsprozesse fokussierenden Ansatz von Margaret Keck und Kathyn Sikkink (1998). Eine Vielzahl von Beiträgen, die diese Grundpositionen politisch reflektieren, sie empirisch-analytisch beantworten, Zwischenpositionen entwickeln, Gegensätze und Widersprüche markieren und den Streit entfalten, füllen diesen Rahmen mittlerweile aus.

Die Breite der Kontroverse, die politische Ernsthaftigkeit des Streits und die damit verbundene Tiefe der Selbstreflektion haben wir als Initiatorinnen der hier präsentierten Diskussion über transnationale feministische Transformationen stets als eine große theoretische und politische Stärke des Feldes geschätzt. Es war uns daher ein besonderes Anliegen, einen Teil dieses Diskurses für unsere Zukunftsbilanz transnationaler Fe- 
minismen lebendig werden zu lassen. Deshalb haben wir verschiedene, für den deutschsprachigen Teil dieser Diskussion entscheidende Personen um einen aktuellen Beitrag zur Debatte gebeten.

Eingeladen zu einem Email-Gespräch über zentrale Fragen zur Gegenwart und Zukunft transnationaler Feminismen haben wir vier Kolleginnen, die drei Generationen des feministischen Streits über feministische Strategien und Erkenntnisperspektiven repräsentieren. Wir haben ihnen schriftlich eine Reihe von Fragen gestellt, die sie nacheinander beantwortet und jeweils an alle Beteiligten gesendet haben, sodass immer alle über die Antworten der Anderen informiert waren und die Möglichkeit zur Reaktion auf andere Statements bestand. Die Namen der beiden älteren Kolleginnen stehen jeweils paradigmatisch für die transnationale Bewegungsdebatte, die aus der Tradition einer feministischen Weltsystemkritik kommt bzw. für die postkoloniale Intervention in die vermeintlichen $\mathrm{Ge}-$ wissheiten der westlichen feministischen Theoriebildung, die eine Generation später erfolgte. Wie wohl kaum eine zweite Person in Deutschland hat Christa Wichterich seit den 1980er Jahren die verschiedenen Phasen transnationaler Feminismen als Aktivistin begleitet und mitgestaltet, als Wissenschaftlerin analysiert und in die feministische Theoriebildung eingeschrieben sowie als Publizistin (keineswegs nur) in die feministische Öffentlichkeit hinein vermittelt (exemplarisch Wichterich 1998; 2003; 2009; 2017). Nikita Dhawan hat seit Beginn der 2000er Jahre die Etablierung postkolonial-queer-feministischer Perspektiven im deutschsprachigen akademischen Kontext maßgeblich vorangetrieben und dabei eine ganze Palette an grundlegenden »Übersetzungsleistungen« erbracht. Zugleich stehen ihre Arbeiten für die kontinuierliche Weiterentwicklung postkolonialer Theoriebildung wie die Kontroverse zwischen materialistisch-intersektionalen und queer-postkolonialen Feminismen (exemplarisch Dhawan 2014; Castro Varela, Dhawan 2015; Dhawan, Castro Varela 2017). Die beiden jüngeren Kolleginnen gehören dagegen einer Generation an, die die Weltfrauenkonferenz in Peking noch nicht bewusst miterlebt hat und deren Feminismus u.a. von den beiden anderen repräsentierten Positionen informiert und geprägt wurde. Dass ihre eigenen Positionen noch nicht im gleichen Maße akademisch etabliert sind wie die der Älteren, versteht sich von selbst. Beide sind gleichwohl seit mehreren Jahren sowohl akademisch als auch aktivistisch mit transnationalem Feminismus befasst, Esther Franke im Bereich Selbstorganisierung über lebensweltliche Anliegen wie Sport oder Ernährung, Radwa Khaled im Bereich Medien und Artivismus (Franke / Wember 2019; Khaled 2017). Wir haben sie eingeladen, gemeinsam eine Position zu formulieren, um ihnen im Kontext mit den sehr 
prominenten älteren Mitschreiberinnen die Möglichkeit zur Zusammenarbeit zu eröffnen. Mit allen vier Kolleginnen haben wir selbst zu unterschiedlichen Zeiten und in unterschiedlichen Kontexten intensiv und mit viel intellektueller Leidenschaft kooperiert und wir haben uns über ihre Beteiligung an diesem Text sehr gefreut.

\section{Uta Ruppert und Tanja Scheiterbauer}

Frage 1: Was ist für Euch das Wichtigste am transnationalen Feminismus? Welches sind mitten in den kumulierenden Krisen des globalen Kapitalismus die wichtigsten feministischen Errungenschaften? Die Weltfrauenkonferenz fand 1995 in dem kurzen historischen Zeitfenster nach dem Ende des Systemantagonismus statt, als die Überzeugung, dass es reelle Möglichkeiten zivilgesellschaftlicher und insbesondere feministischer Einflussnahme auf globale und transnationale Politiken gibt, relativ weit verbreitet war. Gleich, ob wir diesen Ansatz für richtig halten, oder ihn etwa aus postkolonialer Perspektive - zurückweisen, sind die strukturellen Bedingungen für die Umsetzung dessen, was das DAWN-Netzwerk in Peking als "Transformation durch Partizipation" bezeichnete, zweifellos stetig widriger geworden. Zugleich sind starke transnationale Frauenbewegungen mehr oder weniger von der Bildfläche verschwunden. Unsere eigene vorläufige These ist, dass sie in den großen Bewegungen der letzten Jahre wie den Women's Marches oder der sich rasch formierenden transnationalen Solidarität gegen die Ermordung der afrobrasilianischen lesbisch-feministischen Kommunalpolitikerin Marielle Franco Aktualisierungen erfahren, die viele der Veränderungen transnationaler Feminismen in den letzten 25 Jahren widerspiegeln. Wir denken, dass transnationale Feminismen nicht einfach nur an Boden verloren, sondern sich zugleich auch gewandelt und weiterentwickelt haben. Uns geht es mit dieser Frage daher vor allem um die gegenwärtigen Bedeutungen transnationaler Feminismen.

Christa Wichterich: Die Einschätzung des Wichtigsten verändert sich mit transnationalen Feminismen sowie in historischen Kontexten und mit den jeweiligen Perspektiven. Meine Perspektive ist die politischer Praxis und sozialer Bewegungen, und mir scheint wichtig, den transnationalen Feminismus in seiner jeweiligen Zeit zu sehen. Er artikuliert sich in Wellen und Zyklen. In den 1980er Jahren hatte die Transnationalisierung Bedeutung als Versuch, die Drei-Blöcke-Konfrontation von kapitalistischem Westen, postkolonialem Süden und sozialistischem Osten aufzubrechen und den Eurozentrismus in Anerkennung der Vielfalt und der Kritik an den Domi- 
nanz- und Universalitätsansprüchen westlicher Feministinnen durch das neugegründete DAWN-Netzwerk und viele andere Frauenorganisationen aus dem Globalen Süden abzubauen. Damals entstanden auch kontinentale transnationale Vernetzungen wie die Encuentros Feministas in Lateinamerika und der Karibik, Asia Pacific Forum on Women, Law and Development (APFWLD) oder FEMNET in Afrika, die für Positionierungen und Politisierung immens wichtig waren.

Wichtig war außerdem die Erfahrung, dass transnationale zivilgesellschaftliche Organisierung verknüpft mit dem Kampf gegen die Verletzung von Frauenrechten dem globalisierten Kapitalismus etwas entgegensetzen kann. Beispiel 1: der Aufbau transnationalen Widerstands gegen rassistische und eugenische Bevölkerungskontrollpolitik, aber auch gegen westliche Pharmakonzerne (Reichmann/Correa 1994). Beispiel 2: Kämpfe gegen die Ausbeutung von Arbeiterinnen in Exportproduktionszonen und in konzerngesteuerten transnationalen Wertschöpfungsketten, die Produktion und Konsum, Norden und Süden verknüpfen (Marchand/Runyan 2000). Daraus gingen regionale und transkontinentale Frauennetzwerke wie die Clean Clothes Campaign bis zu den Feminists for a Binding Treaty zu Unternehmensverantwortung hervor, die als Solidaritätsprojekte im Sinne von Chandra Mohanty (2003) jahrzehntelang wirkmächtig waren und es noch sind.

In den 1990er Jahren war die Triebkraft der Internationalisierung die Idee der Globalisierung von Menschen-/Frauenrechten, um lokale Themen und Kämpfe in einen Referenzrahmen zu setzen und mit strategischem Essentialismus in internationale und nationale Politik zu intervenieren. Feministinnen aus allen Weltregionen sahen dies - trotz der Kritik an westlichen Universalisierungsansprüchen, trotz vieler Unterschiede und Differenzen, teils mit einer imaginierten Global Sisterhood - als Chance, Gewalt und andere Unrechtserfahrungen mit neuer Legitimation skandalisieren und politisieren zu können (Ruppert 1998; Meyer/Prügl 1999; Marx Ferree/Tripp 2006). Aus politikwissenschaftlicher Perspektive konstruierten die transnationalen Akteur ${ }^{\star}$ innen im Dreieck von Aktivismus, Wissenschaft und Institutionen in der Dynamik zwischen dem Lokalen und der globalen Governance-Ebene (Keck/Sikkink 1998) einen neuen Typus politischer Subjekte (Alvarez 2000) und einen neuen Politiktypus. Der Umzug des Büros von AWID (Association for Women's Rights in Development) von Kanada nach Mexiko signalisierte, dass Impulse zur Transnationalisierung künftig eher vom Globalen Süden als vom Globalen Norden ausgehen sollten. 
Tatsächlich folgte auf die Welle der Transnationalisierung ab den 2000er Jahren eine Gegenbewegung der Lokalisierung und Glokalisierung von Kämpfen, während Intersektionalität und Queer als wichtige Impulse zu komplexerem Denken und Handeln von einer transnationalen Ebene kamen. Die von lateinamerikanischen Feministinnen bei den Weltsozialforen initiierte transnationale Allianz gegen verschiedene Fundamentalismen setzte zukunftsweisende Akzente (Mercosur Feminist Articulation 2002). Afrikanische Feministinnen erarbeiteten eine eigene kontinentale Charter of Feminist Principles for African Feminists (2006).

Heute ist der religiös motivierte, neokonservative, rechtspopulistische Antifeminismus transnational vorzüglich vernetzt, ebenso wie der neoliberale Business- und Karrierefeminismus. Auf Antifeminismus und Homophobie oder Femo- und Homonationalismus muss gewiss jeweils lokal/national reagiert werden, aber es ist auch wichtig zu zeigen, dass es transnationale queer-feministische Antworten gibt und der emanzipations- und transformationsorientierte feministische Transnationalismus nicht zum Verstummen gebracht wurde. Die internationale Kampagne für eine ILO-Konvention zu den Rechten von Hausangestellten war ein Beispiel für eine strategische Nutzung des Intersektionalitätsansatzes. Trotz des Bedeutungsverlusts internationaler Institutionen wie der UNO und der WTO und entsprechend der Global Women's Lobby geben neue Netzwerke wie BRICS Feminist Watch (BFW) im Globalen Süden transkontinental Denk- und Handlungsanstöße. (Queer)Feministische Gruppierungen haben sich mit digitaler Kommunikation und den "sozialen" Medien neue Formate, Artikulationen und Räume angeeignet. \#MeToo verbreitete sich nach dem Schneeballprinzip über Grenzen hinweg und gilt in vielen Ländern als dritte Welle von Frauenbewegungen der jungen Generationen. Zeitgleich wurde mit den Women's Marches zur Verteidigung inklusiver Demokratie gegen autoritäre Übergriffe und den Frauenstreiks die Straße zurückerobert.

Nikita Dhawan: Eine der wichtigsten Errungenschaften des transnationalen Feminismus in den letzten Jahrzehnten war die Überwindung der Idee eines universellen Patriarchats und des Paradigmas der globalen Schwesternschaft ebenso wie die Beschäftigung mit der Frage, wie "Differenzen einen Unterschied machen können« in der globalen Politik (Davis 1983; Crenshaw 1991; Mohanty 2003). Der Fokus auf Intersektionalität hat die Debatten darüber, wie Sexismus, Heteropatriarchat, Rassismus, Antisemitismus, Islamophobie und Transphobie sich gegenseitig bedingen, bereichert, während Kapitalismus und Neokolonialismus weiterhin den größten Teil 
der Weltbevölkerung arm machen und entrechten. Befürworter ${ }^{\star}$ innen eines transnationalen Feminismus betonen, dass ein wachsendes Netzwerk zivilgesellschaftlicher Akteure die Partizipation von Frauen aus dem globalen Süden an der globalen Politik erleichtere. Demgegenüber weisen Kritiker^innen darauf hin, dass die Verschmelzung der lokalen Kämpfe von Frauen mit einer globalen Frauenbewegung hegemoniale feministische Agenden verstärke. Die Universalisierung der Menschenrechte von Frauen beispielsweise hat immer noch mit dem Stereotyp zu kämpfen, genderbedingte Ungleichheit sei allein auf lokale kulturelle Praktiken zurückzuführen. Trotz jahrzehntelanger kontroverser Diskussionen über die Konvention zur Beseitigung jeglicher Form von Diskriminierung gegen Frauen (CEDAW) werden westliche Normen weiterhin als modern und per se emanzipatorisch dargestellt, während die Quelle der Unterdrückung von Frauen aus der Dritten Welt weiterhin hauptsächlich innerhalb der Domäne "primitiver traditioneller" Praktiken verortet wird, wie arrangierte / frühe / erzwungene Heirat, weibliche genitale Beschneidung oder Ehrenmorde. Strategien, die Gewalt gegen Frauen bekämpfen, verstärken Vorstellungen barbarischer und patriarchaler afrikanischer, hinduistischer oder islamischer Traditionen. Die Kulturalisierung und Individualisierung von Frauenrechten lenkt ab von weitreichenderen Fragen globaler struktureller Ungleichheiten, während sie gleichzeitig »Rettungsnarrative« und westliche militärische und humanitäre Interventionen legitimiert (AbuLughod 2013; Spivak 1988).

Esther Franke / Radwa Khaled: Zuallererst wollen wir betonen, dass wir unter den aktuellen Bedingungen nicht von einem transnationalen Feminismus sprechen wollen, sondern verschiedene transnationale Feminismen - im Plural - sehen. Wir stimmen der These zu, dass sich transnationale Feminismen transformiert haben, würden aber in Frage stellen, dass sie an Boden verloren haben.

Die Errungenschaften, die von transnationalen Feminist ${ }^{\star}$ innen auf supranationaler Ebene bis zur Jahrtausendwende erzielt worden sind, von der Anerkennung von Vergewaltigungen als Kriegsverbrechen bis hin zur Infragestellung der globalen Schwesternschaft, sind wichtige Meilensteine (Baksh / Harcourt 2015). Es zeigt sich allerdings, dass die Bemühungen von Femocrats beispielsweise bei der UN nicht zu den gewünschten Ergebnissen führen, auch weil radikalere Ideen und Forderungen in diesen Strukturen eingehegt und abgeblockt werden, da zum Beispiel Tokenism

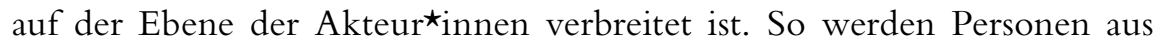


marginalisierten Gruppen zwar individuell mit einbezogen und angehört, allerdings bleiben zu oft strukturelle Änderungen aus (Desai 2005, 2015).

Wir sehen - auch in unserer eigenen politischen Arbeit - eine Verschiebung der Bedeutung der verschiedenen Loci: Es fließt mehr Energie in lokale Bewegungen, die ihre Kämpfe analytisch transnational verorten, ohne direkt supranationale politische Institutionen zu adressieren. Die Bewegungen zeigen sich in unterschiedlichen Orten und Formen, mit unterschiedlichen Praxen, zum Beispiel als Re-Politisierung des 8. März und transnationaler Koordinierung oder als Streiks und Arbeitskämpfe von (migrantischen) Care-Arbeiterinnen ${ }^{\star}$ und kapitalismuskritischen Solidaritätsbewegungen (Arruzza 2018). Diese Perspektive ermöglicht einen Blick auf transnationale Feminismen auch in vermeintlich "kleinen" Aktionen und somit eine Diversifizierung derjenigen, die als transnationale Feminist $\star^{\star}$ innen gesehen werden können.

Diese Veränderungen hängen für uns mit verschiedenen Faktoren zusammen, die sich auch in einem Generationenwechsel kristallisieren. Die Art der Vernetzung der Welt, ihrer Probleme und Krisen, führt zu neuen Diskussionen um Orte des Politischen und des fruchtbaren Konfliktes. Die Generation, die im Neoliberalismus aufgewachsen ist und ihn verkörpert, sucht nach neuen Möglichkeiten des Kampfes gegen das Ohnmachtsgefühl, das sich in unterschiedlicher Weise zeigt und materialisiert. Aus unserer Sicht sind erfolgreiche Kämpfe gegen diese Ohnmacht transnational und feministisch, sie verhandeln die Verhältnisse von Globalem Norden und Süden neu. Haben sich also transnationale Feminismen verschoben, so sind sie dennoch mögliche Lichtblicke in einer vielerorts polarisierten und polarisierenden Welt. Daher wollen wir die Bedeutung der Errungenschaften transnationaler Feminismen, Differenz anzuerkennen und Solidaritäten über diese Differenzen hinweg zu erstreiten, für aktuelle Transformationen betonen.

Frage 2: Welche feministischen Reaktionen auf die gegenwärtigen Krisen des Kapitalismus und der Weltpolitik - von der Care-Krise über die Umwelt- und Nachhaltigkeits-Krise bis zur Krise der Staatlichkeit und der Demokratie - haltet ihr für besonders zukunftsträchtig? Hier interessieren uns nicht nur Eure Perspektiven auf feministische Alternativen transnationaler Politik, sondern auch, wie ihr die Verwobenheit transnationaler feministischer Diskurse und Bewegungspraxen mit den Krisen der Gegenwart einschätzt: Wie wirken Krisenprozesse und Krisenfolgen auf transnationale Feminismen, aber auch umgekehrt, wie verändern transnationale Feminismen Krisendiskurse? Thematische und theoretische 
Fokussierungen lassen wir in dieser Frage bewusst offen, weil uns auch interessiert, welche Ausschnitte aus der Matrix der globalen Krisen ihr diskutieren möchtet.

Christa Wichterich: Auf die systemisch ausgelösten, miteinander verwobenen Krisen reagieren soziale Bewegungen gleichzeitig lokal und international, wie es aktuell etwa in der Klimakrise deutlich wird. Jede Krise fordert Organisierung heraus, die Widerstands- und Alternativpotentiale bündelt und als Versuchsanordnungen konstruiert. Die spezifische Qualität feministischer Ansätze ist, Verbindungen zu Frauenkörpern und Frauenarbeit herzustellen, sprich: zu sozialer Reproduktion und Care. Die Bäuer`innen in La Via Campesina verknüpfen in ihrem feminismo campesino popular seit einigen Jahren die Kämpfe für Ernährungssouveränität, gegen die Gewalt von Landnahme, Industrialisierung und Genmanipulation der Landwirtschaft mit Kämpfen gegen sexualisierte Gewalt und für Souveränität ihrer Körper. Die lateinamerikanische Bewegung gegen $\mathrm{Fe}-$ mizide, Ni una menos, geht davon aus, dass die Gewalt in der Gesellschaft, auch die gegen Natur, über Frauenkörper ausgetragen wird. Ähnlich verbinden die Mitglieder von WoMin im südlichen Afrika ihren Widerstand gegen Bergbau, zerstörerische Ressourcenextraktion durch Investitionen und große Entwicklungsprojekte mit der Forderung nach Anerkennung ihrer tagtäglichen Care-Arbeit, und beharren auf einem Recht, "nein" zum wachstums- und profitfixierten Entwicklungskurs zu sagen. In den Kämpfen des lateinamerikanischen Netzwerks Miradas críticas del Territorio desde el Feminismo gegen den Raubbau an der Natur sind die Verteidigung des eigenen Körpers und die Verteidigung des Landes eng verbunden. Bei ihnen wie auch bei den alle fünf Jahre stattfindenden World March of Women stehen radikale Entwicklungskritik und souveräne (Wieder-)Aneignung der Commons im Zentrum.

Bei der WTO-Ministerkonferenz in Buenos Aires lehnten $2017 \mathrm{mehr}$ als 160 Frauenrechtsorganisationen, vor allem aus dem globalen Süden, eine Erklärung zu "Gender und Handel« ab, die Frauen durch Inklusion in Wertschöpfungsketten, Unternehmertum und Handel wirtschaftlich empowern will. "Pink washing" nennen sie diesen liberalen Gleichheitsansatz und fordern statt der Freihandelsregeln, die ihre lokalen Lebensgrundlagen zerstören, Entwicklungs- und Ernährungssouveränität. Damit verbinden die Herrschafts- und Entwicklungskritiker $\star_{i n n e n}$ auch hier die Mikroebene des Alltags mit der polit-ökonomischen Makroebene im Kampf gegen die Vielfachkrise und die jeweiligen patriarchal-kapitalisti- 
schen Komplexe. Diese Ya-Basta-Bewegungen lassen sich auch als Dekolonisierungsprojekte und anti-imperiale Aneignungsprozesse lesen.

Nikita Dhawan: Jede Krise hat eine Genderdimension und genderspezifische Konsequenzen; trotzdem gibt es keine universellen Lösungen für Probleme, die als global angesehen werden. Es ist sehr wichtig, auf die spezifischen Bedingungen der vielfältigen Krisen in ihren verschiedenen Erscheinungsformen zu achten, aber ebenso auf die spezifischen Taktiken und Strategien, die von denjenigen verwendet werden, die diese Krisen in verschiedenen Weltregionen bekämpfen. Obwohl feministische Initiativen zunehmend hinsichtlich lokaler Kontexte sensibilisiert sind, hat das Konzept der "Interessen von Frauen", die alle Frauen, gleich welcher "Rasse«, Klasse, Religion oder Nationalität, vermeintlich teilen, dazu geführt, dass Patentrezepte für globale Probleme vorgeschlagen werden, die für alle Frauen universell gültig sein sollen. Globale Initiativen gegen Armut und Krieg repräsentieren Frauen aus der Dritten Welt beispielsweise als »hilfsbedürftig" und legitimieren so westliche Interventionen. In dem Ausmaß,

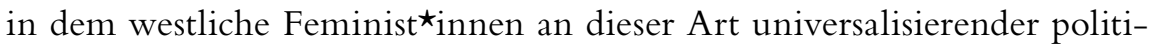
scher Diskurse teilgenommen haben und die Möglichkeit nicht-westlicher Formen der Gendergerechtigkeit abgelehnt haben, haben sie dazu beigetragen, die eurozentrische, verzerrte Wahrnehmung transnationaler feministischer Politik zu verstärken, während sie an einer Form der Solidarität festgehalten haben, die bestehende Hierarchien verstärkt.

Esther Franke / Radwa Khaled: Da die Krisen so vielfältig und verwoben sind und sich gleichzeitig je nach Kontext in unterschiedlicher Ausprägung zeigen, fällt es uns schwer, ein Thema explizit hervorzuheben. Es scheint uns aber wichtig herauszuheben, dass es gerade diese Verwobenheit der Vielfachkrisen ist, die in transnational feministischen Analysen und Praktiken in den Blick genommen und verhandelt wird. Intersektionalität ist dabei ein zentraler Punkt. Eine intersektionale Perspektive wird in akademischen und in politischen Analysen von Unterdrückungsmechanismen eingefordert und vermehrt in politische Praxis übersetzt. So kann das Zusammenwirken verschiedener struktureller Achsen der Differenz in jeweiligen Kontexten und die Verbindungen zu (Re)Produktionen von Ungleichheiten in den Blick genommen werden (u. a. Desai 2015). Jedoch zeigt sich auch hier, dass das Label Intersektionalität nicht immer hält, was

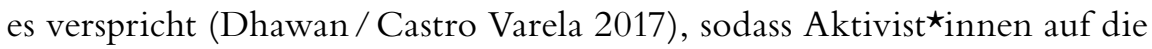
Grenzen dieses Analysegerüsts hinweisen, während sie gleichzeitig für seine konsequente Einbeziehung kämpfen. Auch in akademischer Wis- 
sensproduktion mangelt es an intersektionalen Analysen und nicht zuletzt wird auch hier Intersektionalität als Token in liberalen Projekten benutzt.

Wir beobachten sowohl in unserer akademischen als auch in unserer politischen Arbeit, dass Körper als Orte der Aushandlung von Machtverhältnissen verstärkt ins Zentrum rücken. Dies zeigt sich nicht nur an stärker werdenden Diskursen um Care-Krisen oder Gewalt gegen Frauen, sondern auch am theoretischen Einfluss dekolonialer Perspektiven vor allem aus Lateinamerika, in denen Körper eine zentrale Verbindungsstelle von Strukturen und Individuen repräsentieren (Ribeiro 2017). Auch in der MENA-Region werden Körper als Orte politischen und sozialen Widerstands von Aktivist ${ }^{\star}$ innen und in der postkolonialen Theorie in den Vordergrund gestellt (Hasso, Salime 2016; El Said 2015). Wie verkörpern wir welche Machtverhältnisse und wie lassen sie sich über verkörperte Praxen wandeln? Welche Möglichkeiten bieten Perspektiven auf Körper, um komplexe transnationale und postkoloniale Verhältnisse greifbar zu machen? Die Betonung dieser Perspektiven ermöglicht auch Verschiebungen in Nord-Süd-Verhältnissen. Zwar reproduzieren sich bestimmte Ungleichheiten im globalen neoliberalen Kapitalismus, gleichzeitig zeigen sich jedoch Unterschiede entlang verschiedener Achsen der Differenz, die eine einfache Homogenisierung des Globalen Nordens und Südens unmöglich machen.

Die Krisen betreffen transnationale Feminismen auch insofern, als der Einsatz gegen diese Krisen, deren Drastik und Umfang Feminist $\star_{i n n e n}$ an ihre körperlichen Grenzen bringen. Die Komplexität der Bedrohungen und die vielfachen Formen der Gewalt von verschiedenen Seiten verlangen neue Formen der Selbst- und kollektiven Fürsorge, an denen in feministischen Bewegungen gearbeitet wird und die sich auch auf die Wissenschaft übertragen sollte. Gerade die Angriffe auf Gender Studies an verschiedenen Orten der Welt erhöhen den Druck auf feministische Wissenschaftler ${ }^{\star}$ innen und verlangen nach neuen Formen der transnationalen Solidarität.

Transnationale Feminismen stehen für Bewegung und Transformationen und haben das Potenzial, sich immer wieder neu zu formieren und Analysen und Handlungsoptionen auszuhandeln. Der Mut nicht vor Komplexitäten zurückzuschrecken, ermöglicht ein besseres Verständnis von Verwobenheiten und Betroffenheiten. So können verschiedene Kämpfe zusammengedacht und in ihrer Verbindung mit einer transnational feministischen Perspektive angegangen werden. Daraus entstehen Herausforderungen und Konflikte, aber auch neue Formen der Handlungsmacht (Arruzza 2018, Rajan 2017). 
Frage 3: Die Frage der Solidarität war und ist eine der Kernfragen transnationaler Feminismen. Mit dem Erstarken post- und dekolonialer feministischer Perspektiven, im Zuge der Intersektionalitätsdebatte, aber auch mit den jüngeren feministischen Kämpfen gegen autoritäre, nationalistische und rechtsradikale Politiken und Kräfte hat die Auseinandersetzung um Bedingungen und (Un)Möglichkeiten der Solidarität einmal mehr an Bedeutung gewonnen. Relativ offen scheint uns in diesem Kontext bisher allerdings die Frage der Beiträge aus dem globalen Norden zu Praxen transnationaler Solidarität. Wo seht ihr, von Euren unterschiedlichen Situierungen in akademischen und aktivistischen Kontexten aus betrachtet, Anknüpfungspunkte für transnationale feministische Solidarität aus dem globalen Norden oder ggf. auch präziser aus Deutschland und Europa?

Christa Wichterich: Der Neoliberalismus ist ein Entsolidarisierungs- und Spaltungsprojekt, der Solidarisierung schwieriger, aber auch notwendiger macht. Solidarität heißt, dass ich "euren" Kampf zu meinem mache. Solidarität mit Ungleichen wird umso schwieriger, je stärker die Ungleichheiten zunehmen. Viel diskutiert ist Solidarität z. B. durch ehrenamtliche Arbeit und Alltagshilfe, die den Staat entlastet, `mitleidsökonomisch Ungleichheiten abfedert und damit unbeabsichtigt auch zu ihrer Verfestigung beiträgt. Voraussetzung für feministische Soliarbeit ist, Diskurse und Bewegungspraktiken zu enthierarchisieren, zu dekolonisieren und sie gleichzeitig zu politisieren, um sich gegen politische und ökonomische Vereinnahmung zu sperren. Trotz aller identitätspolitischen und postkolonialen Furcht vor einem homogenisierenden Wir und bevormundenden Kollektivsubjekten gibt es keine Alternative dazu, Solidarität immer wieder als Versuchsanordnung $\mathrm{zu}$ erproben und Intersektionalität $\mathrm{zu}$ einem strategischen Prinzip zu machen. Europäische Feministinnen stellen als Ausgangspunkt für Solidarisierung häufig die Frage: Was erwartet Ihr von uns, was wünscht Ihr, wie wir uns verhalten?

Ohne diesen Explorationswillen gäbe es im Kontext von Migration, Flucht und Asyl keine solidarischen Gemeinschaftsgärten, keine solidarischen Städte, die unter Handlungsdruck und den gegebenen Bedingungen Nischen schaffen, aber soziale und politische Transformation ansteuern und nicht nur Notsituationen verwalten. Und es gäbe keine Frauenstreiks. Ausgehend von den Anti-Femizid-Kampagnen in Lateinamerika und den Protesten gegen das Abtreibungsverbot in Polen wurden Streiks als politisches Mittel der Verweigerung eingesetzt, zunächst in Einzelaktionen in Argentinien, Mexiko, Chile und Spanien, seit 2017 immer mehr als transnationale Welle (Gago et al 2018). Streiks werden derzeit vielerorts 
als zukunftsfähigste Kampfform gesehen, mit den größtmöglichen Sichtbarkeitseffekten und Potentialen zu feministischer Klassenbildung (Aruzza 2018).

Eine solche Versuchsanordnung auf Grundlage der "neuen Welle des militanten feministischen Aktivismus" und eine transnationale Debattengrundlage ist auch das Manifest "Feminismus für die 99 Prozent« (Aruzza / Bhattacharya / Fraser 2019), das mit antikapitalistischem Fokus Spaltungen von Geschlecht, Ethnie, Hautfarbe, Behinderung, Sexualität sowie den Gegensatz von Identitäts- und Klassenpolitik überwinden will.

Nikita Dhawan: Mein Problem mit dem transnationalen Feminismus ist, dass er es versäumt, sich ernsthaft mit jenen historischen Prozessen zu beschäftigen, durch die bestimmte Individuen sich in einer Situation wiederfinden, aus der sie globale Solidarität anstreben können. Die Entfernung zwischen denen, die Solidarität "austeilen" und denen, die einfach als "Opfer von Unrecht« und somit als "Empfänger» kodiert werden, bleibt ein Charakteristikum historischer Gewalt (vgl. Spivak 2008). Die fehlende Wechselseitigkeit und die Distanz zwischen diesen beiden Seiten ist der Beleg dafür, dass der dekolonisierende Feminismus gescheitert ist. Ohne eine Auseinandersetzung mit der ungleichen Verteilung von Handlungsmacht auf globaler Ebene und deren Überwindung wird transnationale feministische Politik weiter als Alibi für den imperialen Feminismus fungieren.

Esther Franke / Radwa Khaled: Wir verstehen Solidarität nicht als ein Set von Praxen, die vom geographischen Norden zum geographischen Süden fließen. Die Trennung Norden-Süden ist politisch und analytisch wichtig, um historische und gegenwärtige Formen der kapitalistischen und anderweitigen Ausbeutung politisch benennen und wissenschaftlich analysieren zu können. Die Verbindungen sind allerdings komplex und nie unidirektional (Bueno-Hansen / Falcón 2017, Carty / Mohanty 2015). Auch Solidaritäten dürfen nicht in dieser Dichotomie verharren und wir beobachten an verschiedenen Stellen, dass Praxen der Solidarität Nord-Süd-Grenzen durchqueren und dazu beitragen, dieses Verhältnis neu zu denken. Die Eingewobenheit in Machtverhältnisse im neoliberalen Kapitalismus bringt nicht nur neue Differenzen, sondern auch neue Bündnisse hervor. In unserer politischen Arbeit merken wir, wie Verschiebungen im Umgang mit Differenzen und Solidarität stattgefunden haben. Verbindungen werden aufgrund von Themen, Haltungen und Kämpfen gesucht, die verwobene Formen des Aktivismus schaffen. Sichtbar wird dies zum Beispiel in den 
Bemühungen um Dekolonialisierung, in denen unterschiedliche Privilegien und Loci des Sprechens ausdifferenziert und daraus Möglichkeiten des Handelns ausgearbeitet werden. Dass dieser Prozess nicht frei von Konflikten und auch ein stetiges Ringen um die Bedeutung von Feminismus ist, steht solidarischen Praxen nicht im Wege, sondern ist eher Teil davon. Hier ist ein Blick auf konkrete Aushandlungen von Solidarität in Interaktionen und auf die affektiven und körperlichen Dimensionen dieser Konflikte und Verbindungen spannend. Gerade in alltäglichen Praxen sehen wir hier Chancen (Franke / Wember 2019). Transnationaler Feminismus muss auch mit der Alltagswelt zusammen gedacht werden, etwa den Themen Ernährung, Wohnen oder Sport - auch dies sind Orte, an denen transnationale Feminismen eine Rolle spielen und wo transnational feministische Praxen gefunden werden können.

Was kann Solidarität konkret bedeuten? In einem Zeitalter, in dem Menschen und Bewegungen durch die neuen sozialen Medien verbunden sind, sind unmittelbare Solidarisierungen zeitlich leicht möglich. Allerdings kommt es auch schnell zu einem Aktivismus, der sich auf den Raum der sozialen Medien fokussiert, sowie zu kürzeren Zyklen der Auseinandersetzungen mit den Themen. Damit verbunden sind Fragen zur Bedeutung der physischen Begegnung für transnationale Feminismen. Angesichts der Tatsache, dass analoge sowie digitale Begegnungs- und Handlungsräume und die in ihnen entstehenden radikaleren Möglichkeiten der Solidaritäten eingehegt werden können durch die neoliberale Logik der social Media Plattformen, der "Funding-Logik» von Stiftungen und anderer Geldgeber sowie der sich verengenden Möglichkeiten des vollständig autonomen Organisierens und Handelns. Universitäten sehen wir als besonders fruchtbare Räume. Sie sollten nicht nur als ein kontinuierlich neoliberalisierter Betrieb gesehen werden, sondern auch als ein vernetzter Handlungsort, an dem in der Wissenschaftspraxis und darüber hinaus trotz prekärer Bedingungen - Räume für Solidaritäten geschaffen werden können. Dazu gehört auch, die Dichotomien von epistemisch versus politisch und materiell versus diskursiv in der feministisch-dekolonialen Wissenschaftspraxis permanent zu dekonstruieren.

Frage 4: Zum Schluss möchten wir die Frage stellen, welche Aspekte unserer gemeinsamen Diskussion Euch als besonders zukunftsrelevant erscheinen. Welche Argumente müssten wissenschaftlich oder/und politisch vertieft werden? Welches sind Eurer Meinung nach die wichtigsten Arenen und ggf. auch Methoden zukünftiger Auseinandersetzung? Welche weiteren oder auch ganz anderen Fragen würdet ihr stellen? 
Christa Wichterich: Aufgrund des anti-feministischen Rollbacks und neoliberaler Vereinnahmungen besteht derzeit ein vehementes Interesse, verschiedene Feminismen und Kämpfe miteinander zu verknüpfen, identitätspolitische Fragmentierungen zu überwinden und gezielt strategische Allianzen einzugehen. Neben herrschafts-, rassismus- und homogenisierungskritischem Wissen brauchen wir Transformations- und Dekolonisierungswissen, z.B. wie Intersektionalität von einer Analysekategorie in eine polit-strategische Kategorie umgesetzt werden kann. Patriarchat, Kapitalismus und Kolonialismus müssen in ihrer Verwobenheit gedacht, umverteilungs- und identitätspolitische Strategien verknüpft werden. Das soll auch der neoliberalen Dethematisierung der Kategorie Klasse als Ursache von Ungleichheiten und Ungerechtigkeiten entgegenwirken (Wolter/Wischnewski 2019). Auch die vielen alternativen Wirtschaftsund Lebensweisen, die Feminist $\star_{i n n e n}$ mitgestalten, müssen identifiziert und in konstruktive Beziehungen gesetzt werden, die Emanzipation und Transformation verbinden. Das heißt, dass sowohl feministische politische Ökonomie als auch feministische politische Ökologie analytisch und normativ weiterentwickelt werden müssen.

Migration und Flucht setzen einen neuen politischen Rahmen für feministische Forschungsfelder, Solidaritäten und Organisierung auf dem Kontinuum zwischen Globalem und Lokalem und in hybriden Räumen. Zudem fordern außer dem massiven Anti-Feminismus aktuelle transnationale Entwicklungen wie die alarmierende Militarisierung, ja Kriegstreiberei, die Digitalisierung von Arbeit und Alltag und die Bioökonomie aktuelle feministische Antworten mit glokaler Perspektive heraus. Dabei müssen unter den Bedingungen von Hate Speech and Fake News, aggressiven Versuchen, Gender Studies zu diskreditieren und auszuschalten, und An-

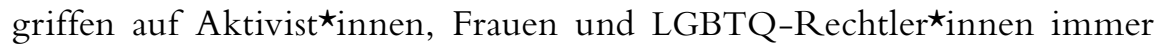
wieder neue Formen und Formate von Organisierung und Solidarisierung öffentlich sichtbar erkundet werden, um die gewonnenen Räume und Instrumente zu erhalten. Der Fokus auf Identitäten, Resonanzen und Selbstsorge darf den analytischen und transformatorischen Blick auf kapitalistische und koloniale, patriarchale und rassifizierte Herrschaftsstrukturen nicht trüben (Wichterich 2016).

Nikita Dhawan: Genderbasierte Gewalt wird weiterhin von hegemonialen Kräften als Waffe benutzt, um MigrantInnen und postkoloniale Gesellschaften als inhärent gewalttätig und frauenfeindlich zu stigmatisieren, während Migrantinnen und Frauen in der Dritten Welt als »hilflose Opfer« ihrer Kulturen und Gesellschaften konstruiert werden. Angesichts westli- 
cher imperialistischer Praktiken besteht ein Risiko, dass jede Diskussion von genderbasierter Gewalt innerhalb von migrantischen Gemeinschaften oder in postkolonialen Gesellschaften strategisch kooptiert wird, um Rassismus und Militarisierung im Namen des "Schutzes" vor genderbasierter Gewalt zu perpetuieren. Die wachsende Verbreitung von Diskursen über Gewalt behindert gleichzeitig den Kampf gegen genderbasierte Gewalt. Die transnationale feministische Forschung scheint immer noch Probleme damit zu haben, Allianzen gegen genderbasierte Gewalt zu entwickeln, welche eine Plattform für zukünftige feministische Projekte liefern könnten. Wie können neue Arten kollektiver Handlungsmacht entstehen, die genderbasierte Verletzlichkeit als Quelle politischer Handlungsmacht nutzen, um genderbasierte Ungerechtigkeit auf globaler Ebene zu bekämpfen? Wie können Probleme sexueller Gewalt und genderbasierter Ungerechtigkeit bekämpft werden, vor allem im globalen Süden, ohne orientalistische und eurozentrische Impulse im globalen Norden zu verstärken? (Castro Varela / Dhawan 2016)

Esther Franke / Radwa Khaled: Zentral scheint uns, die Debatten um die Dekolonisierung von Feminismen weiterzuführen und zu vertiefen. Welche Praxen tragen dazu bei? Welche neuen Räume des Austausches - politisch und akademisch - müssen geschaffen und gesucht werden? Wo sind diese vielleicht bereits vorhanden und können als solche erkannt werden? Wo können wir nach Praxen von transnationaler feministischer Solidarität suchen? Welche Rolle spielen hier lebensweltliche Bereiche? Neue Formen und Haltungen zu Widerstand und Transformation, wie beispielsweise die Betonung von life oder liveability in feministischen Bewegungen in der Türkei (Franke / Khaled 2019) scheinen uns interessant, genauso der stärkere Blick auf Körper und Affekt in Bezug auf transnationale Machtverhältnisse und Solidarität. Welche Formen und Verständnisse von Care, die auch Sicherheit und Schutz vor Gewalt mit einbeziehen, können und sollten weitergedacht werden?

Wie und wo können transnationale Handlungsfähigkeiten in unserer komplexen Welt gestärkt werden ohne Machtungleichheiten zu reproduzieren? Welche (neuen) Rollen können politische Institutionen spielen? Welche Rolle kommt Staaten zu - in Ansprache, Kritik und Transformation? In welche Richtungen sollen sich transnationale Feminismen in einer sich polarisierenden und von Anfeindungen und Konfrontationen geprägten Welt transformieren?

Zentral sind auch epistemisch politische Fragen. Welches Wissen wird für wen produziert und auf welche Art? Die Frage, wie gerade feministi- 
sche Wissenschaft Wissen produzieren kann, das für Bewegungen nützlich ist, und sich gleichzeitig in der umkämpften Welt der Wissenschaft mit all den Angriffen und Anfeindungen behaupten kann, wird immer wichtiger. Ist auch in den Gender Studies Dekolonisierung wirklich erwünscht und angestrebt? Wenn ja, was hieße dies auch praktisch?

\section{Literatur}

Abu-Lughod, Lila (2013): Do Muslim Women Need Saving? Cambridge.

Alexander, Jacqui / Mohanty, Chandra (Hrsg.) (1997): Feminist Genealogies, Colonial Legacies, Democratic Futures. New York, London.

Alvarez, Sonia (2000): Translating the Global: Effects of Transnational Organizing on Local Feminist Discourses and Practices in Latin America. Meridians 1 (1), 29-67.

Aruzza, Cinzia (2018): From Women's Strikes to a New Class Movement: The Third Feminist Wave. In: Viewpoint Magazine (3.12.2018). https://www.viewpointmag.com/ 2018/12/03/from-womens-strikes-to-a-new-class-movement-the-third-feministwave/ (10.02.2020)

Aruzza, Cinzia/Bhattacharya, Tithi/Fraser, Nancy (2019): Feminismus für die 99 Prozent. Ein Manifest. Berlin.

Baksh, Rawwida / Wendy Harcourt (2015): Introduction. Rethinking Knowledge, Power, and Social Change. In: Baksh, Rawwida/Harcourt, Wendy (Hrsg.): The Oxford Handbook of Transnational Feminist Movements. New York, 1-52.

Bueno-Hansen, Pascha/Falcón, Sylvanna M. (2017): Indigenous / Campesina Embodied Knowledge, Human Rights Awards, and Lessons for Transnational Feminist Solidarity. In: McLaren, Margaret A. (Hg.): Decolonizing Feminism. Transnational Feminism and Globalization. London, 57-82.

Carty, Linda E./Mohanty, Chandra T. (2015): Mapping Transnational Feminist Engagements. Neoliberalism and the Politics of Solidarity. In: Baksh, Rawwida/Harcourt, Wendy (Hrsg.): The Oxford Handbook of Transnational Feminist Movements. New York, 82-115.

Castro Varela, María do Mar/Dhawan, Nikita (2015): Postkoloniale Theorie: Eine kritische Einführung. Bielefeld.

Castro Varela, María do Mar/Dhawan, Nikita (2016): Die Migrantin retten!? Zum vertrackten Verhältnis von Geschlechtergewalt, Rassismus. und Handlungsmacht. In: Österreichische Zeitschrift für Soziologie 41 (3), 13-28.

Charter of Feminist Principles for African Feminists. http://www.africanfeministforum. com/feminist-charter-introduction/ (23.01.2020)

Crenshaw, Kimberlé (1991): Mapping the Margins: Intersectionality, Identity Politics, and Violence against Women of Color. In: Stanford Law Review 43 (6), 1241-1299.

Davis, Angela (1983): Women, Race and Class. New York.

Desai, Manisha (2015): Critical Cartography, Theories, and Praxis of Transnational Feminisms. In: Baksh, Rawwida/Harcourt, Wendy (Hrsg.): The Oxford Handbook of Transnational Feminist Movements. New York, 116-130. 
Desai, Manisha (2005): Transnationalism. The Face of Feminist Politics Post-Beijing. In: International Social Science Journal 57 (184), 319-330.

Dhawan, Nikita (Hrsg.) (2014): Decolonizing Enlightenment: Transnational Justice, Human Rights and Democracy in a Postcolonial World. Opladen / New York.

Dhawan, Nikita / Castro Varela, María do Mar (2017): "What Difference Does Difference make?: Diversity, Intersectionality and Transnational Feminist Politics."In: Dhawan, Nikita (Hrsg.): Difference that makes no Difference. The Non-Performativity of Intersectionality and Diversity. Special Issue. Wagadu. A Journal of Transnational Women's and Gender Studies 16, 11-39.

El Said, Maha / Meari, Lena / Pratt, Nicola (Hrsg.) (2015): Rethinking Gender in Revolutions and Resistance. London.

Franke, Esther M. / Wember, Carla (2019): , The Power of Food to Bring People Together and Create Common Ground - Affektive Dissonanz und transnationale Solidarität in einem Ernährungsnetzwerk. In: Femina Politica 2, 94-107.

Franke, Esther M. / Khaled, Radwa (2019): Feminismus im Plural. Bericht über die Colloquiumsreihe »Feminismen des Globalen Südens« des Cornelia Goethe Centrums an der Universität Frankfurt. In: Iz3w 370. https://www.iz3w.org/zeitschrift/ausgaben/370_ gefaengnisse/khaled (03.02.2020)

Gago, Verónica / Aguilar, Raquel, Gutiérrez / Draper, Susana / Diáz, Mariana Menéndez / Bardet, Marie / Rolnik, Suely (2018): 8M - der große feministische Streik. Wien.

Grewal, Inderpal / Kaplan, Marion (Hrsg.) (1994): Scattered Hegemonies. Postmodernity and Transnational Feminist Practice. Minneapolis/London.

Hale, Angela (Hrsg.) (1998): Trade Myths and Gender Reality: Trade Liberalisation and Women's Lives. Uppsala.

Hasso, Francis S. / Salime, Zakia (Hrsg.) (2016): Freedom without Permission: Bodies and Space in the Arab Revolutions. Durham.

Khaled, Radwa (2017): Künstlerische Alltagspraktiken als Politik. Perspektiven der (Nicht-) Bewegungsforschung auf Ägypten. In: Peripherie 1, 29-51.

Keck, Margaret/Sikkink, Kathryn (1998): Activists Beyond Borders. Ithaka.

Marchand, Marianne/Runyan, Anne Sisson (2000): Gender and Global Restructuring: Sightings, Sites, and Resistances. Abingdon / New York.

Marcosur Feminist Articulation (2002): your mouth is fundamental against fundamentalisms. Montevideo.

Marx Ferree, Myra / Tripp, Aili Mari (2006): Transnational Feminisms: Women's Global Activism and Human Rights. New York.

Meyer, Mary K. / Prügl, Elisabeth (Hrsg.) (1999): Gender Politics in Global Governance. Lanham.

Mohanty, Chandra Talpade (Hrsg.) (2003): Feminism without Borders. Decolonizing Theory, Practicing Solidarity. Duke.

Rajan, Hamsa (2017): The Ethics of Transnational Feminist Research and Activism. An Argument for a More Comprehensive View. In: Signs 43 (2), 269-300.

Reichmann, Rebecca / Correa, Sonja (1994): Population and Reproductive Rights. Feminist Perspectives from the South. London.

Ruppert, Uta (Hrsg.) (1998): Lokal bewegen - global verhandeln. Internationale Politik und Geschlecht. Frankfurt/M. / New York.

Ribeiro, Djamila (2017): O que é lugar de fala? (What is the standpoint of speech?). Belo Horizonte. 
Spivak, Gayatri Chakravorty (1988): Can the subaltern speak? In: Nelson, Cary/ Grossberg, Lawrence (Hrsg.): Marxism and the Interpretation of Culture. Urbana, 271-313.

Spivak, Gayatri Chakravorty (2008): Righting Wrongs - Unrecht richten: Über die Zuteilung von Menschenrechten. Zürich.

Wichterich, Christa (2017): Geschlechtergerechtigkeit zwischen neoliberalem Empowerment und post-kolonialer Kritik. In: Estermann, Josef (Hrsg.): Das Unbehagen an der Entwicklung. Eine andere Entwicklung oder anders als Entwicklung? Aachen, 63-77.

Wichterich, Christa (2016): Feminisms on the Move. Against the Backlash, Towards New Solidarities. Berlin. https://library.fes.de/pdf-files/iez/12828.pdf (13.02.2020)

Wichterich, Christa (2009): gleich, gleicher, ungleich. Paradoxien und Perspektiven von Frauenrechten in der Globalisierung. Königstein / Taunus.

Wichterich, Christa (2003): Femme Global. Die Globalisierung ist nicht geschlechtsneutral. Hamburg.

Wichterich, Christa (1998): Die globalisierte Frau. Berichte aus der Zukunft der Ungleichheit, Reinbek bei Hamburg 1998 (The Globalized Woman, Reports from a Future of Inequality, London 2000. La femme mondialisée, Actes Sud, Paris 1999).

Wolter, Kerstin/Wischnewski, Alex (2019): Eine feministische Internationale. Wie sich Frauen ${ }^{\star}$ über Grenzen hinweg organisieren. In: LuXemburg 1. https://www.zeitschriftluxemburg.de/eine-feministische-internationale-wie-sich-frauen-ueber-grenzen-hin weg-organisieren/ (09.02.2020) 